\title{
STATIC VERSUS DYNAMICAL COSMIC-RAY HALOS
}

\author{
FRANK C. JONES \\ Laboratory for High Energy Astrophysics, Code 665 \\ NASA/Goddard Space Flight Center \\ Greenbelt, MD 20771, USA
}

\begin{abstract}
The dynamical halo of the Galaxy offers a natural explanation for the form of the variation of cosmic-ray path length with energy. The variation above $1 \mathrm{GeV}$ per nucleon can be understood as due to the variation of the diffusion coefficient, and hence the resident time in the galaxy, with energy. The flattening of the curve below $1 \mathrm{GeV}$ per nucleon is seen to mark a transition to a convection dominated regime where the variation of the diffusion coefficient is no longer a determining factor. It is possible that the random motion of the cosmic rays about the galaxy that prevents us from seeing their sources in a clear manner may enable us to extract information about the galaxy at large and learn something about its large scale motions.
\end{abstract}

\section{INTRODUCTION}

It was pointed out by Shklovsky (1952) that the observed high latitude distribution of non-thermal radio emission could be explained by postulating an extensive halo of radio emissivity surrounding the disk of the Galaxy. Pikel'ner (1953) suggested that a gaseous halo with a magnetic field of $\approx 3 \times 10^{-6}$ gauss would be needed to retain the particles responsible for the emission. Soon ideas of this sort of galactic halo had permeated the thinking of those concerned with the problem of the propagation of cosmic rays in the Galaxy (Bierman and Davis, 1958.)

The notion of storage of cosmic rays in a volume larger than the galactic disk was attractive because it gave a natural answer to the question of why the radiation was so isotropic. Furthermore, it offered an explanation for the fact that the combination of mean age of the cosmic rays obtained from the study of unstable isotopes and the mean path length from the primary to secondary ratios yielded a matter density over the particles lifetime of 0.06 particles $\mathrm{cm}^{-3}$ while the density in the galactic disk was believed to be about 1 particle $\mathrm{cm}^{-3}$.

The notion of a large, static, low density region surrounding the galaxy that was able to trap a significant number of energetic particles proved, however, to 
be untenable. Several authors showed that, if nothing else, the energetic cosmic rays themselves had more than enough pressure to blow away this galactic halo or corona. If such a halo existed it would have to be a dynamical one, with a constant galactic wind constantly replenished from the higher density disk region of the galaxy.

In the following we shall examine the ways in which the dynamical nature of the halo will manifest itself in the properties of the cosmic-ray electrons and nucleons and try to determine what measurements of these particles can tell us about the nature of the halo of our galaxy.

\section{COSMIC RAY ELECTRON HALO}

The primary manner in which a galactic halo that traps or holds energetic electrons manifests itself is through the region of radio emission surrounding the galaxy. As one might imagine, the difference between a dynamic halo with outflow and a static one could be rather subtle in such a circumstance, manifesting itself only through the detailed manner in which the electron spectrum, and hence that of the radio emission, changes with position in the halo.

A very detailed development of the theory of electrons in an outflowing halo has been presented in a series of papers by Lerche and Schlickeiser (1980, 1981a-c)

Werner (1988) has presented results of observations of the edge on galaxy NGC4631 at several different frequencies and concluded that the predictions of Lerche and Schlickeiser were confirmed. The confirmation was based on the observation of a break in the radio spectrum (reflecting a corresponding break in the electron spectrum.)

\subsection{A Simple Theory}

Since the expressions of Lerche and Schlickeiser are quite complicated it would be useful to derive expressions which, although greatly simplified, can show more clearly the basic difference between the electron spectra to be expected in the dynamical and static halos. The difference arises from the different time behavior of diffusive and convective transport which are working against the continuous change of the electron spectrum produced by the synchrotron radiation process which is responsible for the radio emission itself.

First we should note that electrons that are emitting radiation via the synchrotron process (or inverse Compton scattering as well) lose energy at a rate proportional to the square of their energy, ie.

$$
\frac{d E}{d t}=-b E^{2}
$$


If we integrate equation (1) from an initial energy $E_{1}$ to a final energy $E_{2}$ we obtain

$$
b t=\frac{1}{E_{2}}-\frac{1}{E_{1}} \text {. }
$$

and setting $E_{1}$ equal to $\infty$ we obtain

$$
E_{c}=\frac{1}{b t} .
$$

Equation (2) shows that after a time $t$ no particles can remain above the critical energy $E_{c}$. In other words any injection spectrum whatsoever will exhibit a cutoff at $E_{c}$ after a time $t$.

A power law spectrum of the form

$$
N(E, t=0)=A E^{-q}
$$

will have the form at time $t$

$$
N(E, t)=A E^{-q}(1-b E t)^{q-2} .
$$

From this we can see that a reasonable approximation to the correct spectrum after a time $t$ has passed is to multiply an injection spectrum of any form by a step function $U(E, b t)$ that has a value of unity for $E<1 / b t$ and is zero for $E>1 / b t$.

With this approximation it is easily seen that if we have a distribution of particle ages rather than a discrete time after injection our step function $U(E, b t)$ should be replaced by

$$
U(E)=\int_{0}^{\infty} f(t) U(E, b t) d t=\int_{0}^{1 / b E} f(t) d t
$$

where $f(t)$ is the age distribution function.

\subsection{A Halo Model}

Before proceeding to look at the age distributions that are produced by static and dynamical halo models we should first see what a theoretician's galaxy looks like. 


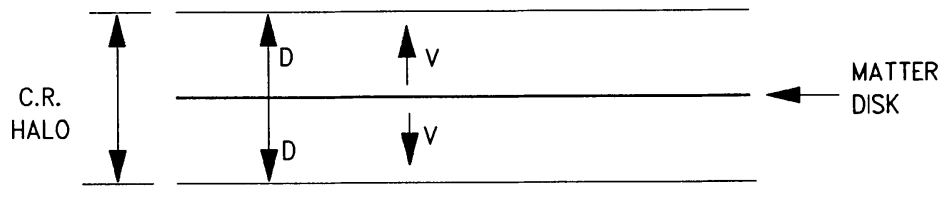

Figure 1. Cosmic-Ray Theoretician's View of a Galaxy

Figure 1 shows that the essential components of the galaxy are the disk, usually considered to contain all or almost all of the matter, and the halo which is flowing away from the disk on both sides with a (possibly variable) velocity $V$. The matter in the halo is considered to be significant only in that it carries the irregular magnetic fields which convect the energetic particles and through which they diffuse. In most cases the extent of the disk and halo in directions parallel to the disk is considered to be infinite thus reducing the problem to one dimension.

If particles are released at the position of the disk $\dagger$ they are convected a distance $x$ out into the halo in a time $t$ with $x=V t$ where $V$ is the halo wind speed. While their mean position is being convected the particles are also diffusing about this center so that their density as a function of $x$ and $t$ is given by

$$
N(x, t)=\frac{\exp \left(\frac{-(x-V t)^{2}}{2 \kappa t}\right)}{\sqrt{2 \pi \kappa t}}
$$

where $\kappa$ is the diffusion coefficient of the particles in the flowing halo magnetic fields.

Characteristic Length and Time At this point it would be advisable to note that there are two parameters in the problem $(\kappa$ and $V)$ that have different dimensions. This means that certain characteristic dimensions can be formed from these parameters. A more physical way of looking at this is to realize that in diffusion a particle travels a distance $x$ in a time $x^{2} / \kappa$ whereas in convection it travels the same distance in a time $x / V$. There is clearly a characteristic distance given by $x_{c}=\kappa / V$ for which these times are equal and the corresponding characteristic time is $t_{c}=\kappa / V^{2}$. For distances shorter than $x_{c}$ diffusion is the quickest way to travel and we can say that we are in a diffusion dominated regime. However if the lengths of interest are greater than $x_{c}$ convection is faster than diffusion and we are in a convection dominated regime.

$\dagger$ All models that I am familiar with assume that the energetic cosmic rays are produced in the disk portion of the galaxy, presumably by supernova related phenomena 
Obviously if the size of the halo, $D$ is smaller than $x_{c}$ the entire halo will be diffusion dominated and it will be very difficult to talk in a meaningful way about a dynamical halo as far as the cosmic ray particles are concerned. We, therefore, require for a dynamical halo in the energetic particle sense that $x_{c}<D$ (actually we really need $x_{c} \ll D$ for convective effects to be pronounced enough for unambiguous observation.)

Employing the characteristic length and time that we have defined we may express equation (5) in dimensionless units as

$$
N\left(x^{\prime}, t^{\prime}\right)=\frac{1}{x_{c} \sqrt{\pi}} \exp \left(\frac{-\left(x^{\prime}-t^{\prime}\right)^{2}}{2 t^{\prime}}\right)
$$

where $x^{\prime}=x / x_{c}$ and $t^{\prime}=t / t_{c}$.

Time Distribution for $x^{\prime} \gg 1$ If we look out in the halo a distance that is large compared with the characteristic distance $x_{c}$ we find that convection dominates at all times and a pulse of width $\approx x^{\prime-1 / 2}$ is swept by us at a time $t^{\prime}=x^{\prime}$ as shown in Figure 2.

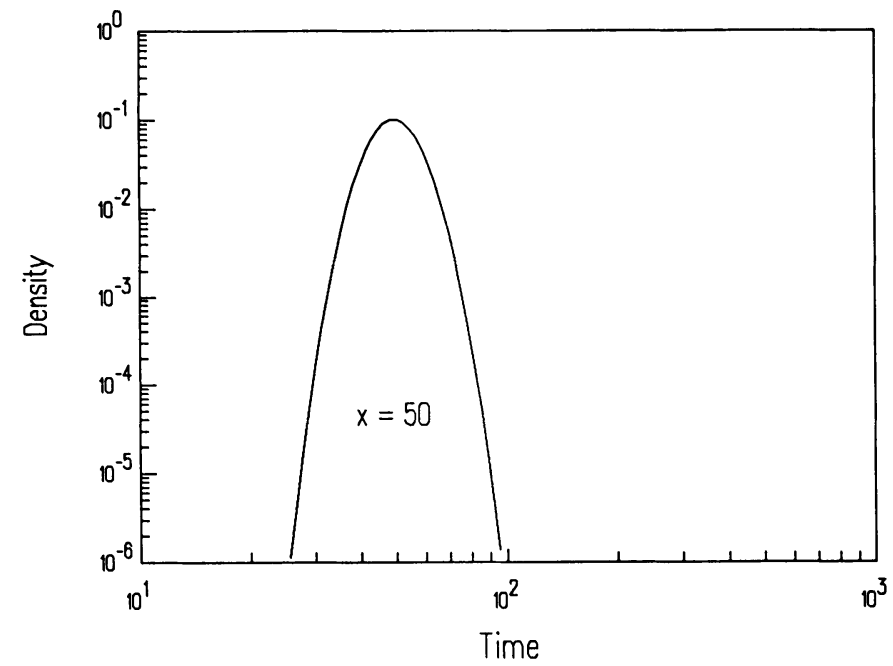

Figure 2. Time Dependance of Density for $x^{\prime} \gg 1$

Time Distribution for $x^{\prime} \ll 1$

In Figure 3 we see plotted the density of particles as a function of time (or age) at a position well inside the characteristic length for the outflowing halo we see that the time profile looks exactly like a purely diffusive one until we reach 


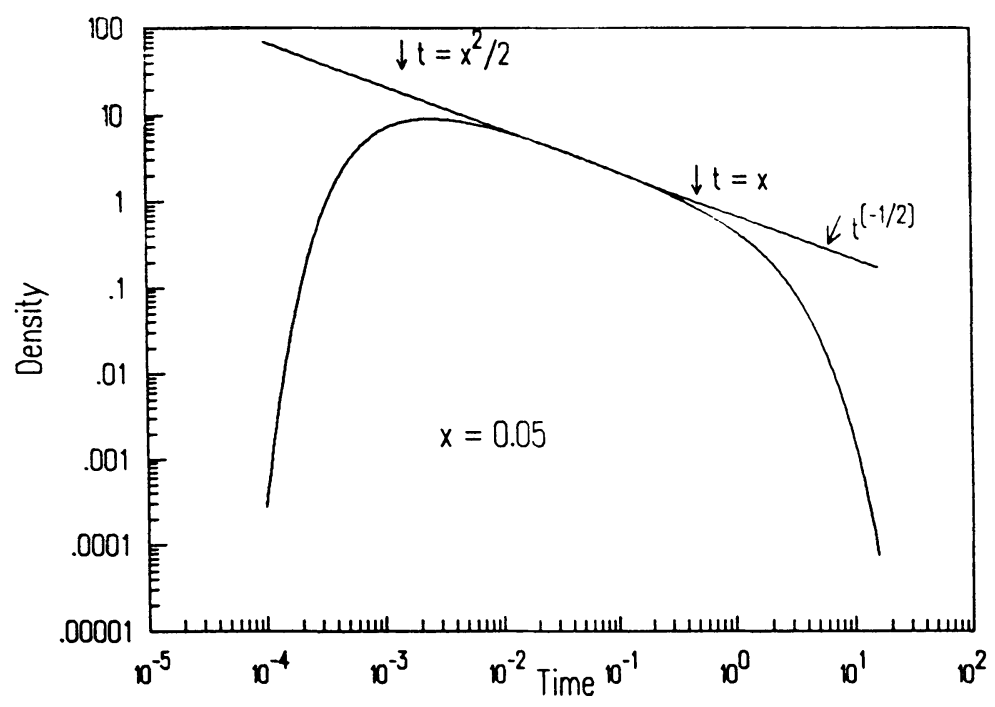

Figure 3. Time Dependance of Density for $x^{\prime} \ll 1$

a time when convection effects can reach the observation point ie. when $t^{\prime}=x^{\prime}$. After this time the particle density no longer decays with a $t^{\prime-1 / 2}$ time dependance that is characteristic of diffusion but instead is swept away with a $\exp (-V t)$ time dependance typical of convection. This is strictly true only in the one dimensional, infinite extent halo; a higher dimensionality or an absorbing boundary will change this as we will see later.

We note that in this case there are two times of importance $t^{\prime}=x^{\prime 2} / 2$ at which time the density rises sharply from zero and $t^{\prime}=x^{\prime}$ at which time the density drops rapidly to zero again with a $t^{1-1 / 2}$ dependance in between.

We can now see that for the convection dominated case there is essentially one time for each position in the dynamical halo and the electron spectrum at that point is unchanged below the characteristic energy $E_{c}$ and is rather sharply cut off above that energy. The time is given by the relation $t=x / V$ and therefore $E_{c}=V / b x$, the characteristic energy is lower the further out in the halo one looks.

On the other hand, whenever one looks in a region where diffusion dominates the two characteristic times produce a somewhat different spectrum. It is straightforward to see that once again the spectrum is unchanged from its injection form for energy below $E_{c}=V / b x$. However, above that energy the spectrum will be multiplied by a "filter" function proportional to $E^{-1 / 2}$ or, if it is a power law spectrum, steepened by a one half power. The cutoff occurs at an energy $E_{c 2}=2 \kappa / b x^{2}$. So we see that the signature of diffusion is the extension of the cutoff with a half power steepening to an energy characteristic of the diffusion time scale. 
If there is no outflow at all (a static halo) the half power steepening will continue down to the lowest energy in the injection spectrum. Such a condition would be hard to distinguish from the convection dominated case were it not for the fact that the cutoff in the spectrum varies with distance out into the halo by a different power of distance in the two cases. In the pure diffusion case the cutoff energy is inversely proportional to the square of the distance while in the convection dominated case it is inversely proportional to the distance.

It should also be noted that the bend in the spectrum is a signature of convection only in the case of a one dimensional, infinite extent halo. Any deviation from this situation such as an absorbing boundary or a transition to three dimensional diffusion will produce a characteristic time, $t_{c}$ such that for $t>t_{c}$ the time profile drops off faster than $t^{-1 / 2}$ and produces such a bend in the spectrum. In this case the variation of the energy of the bend with distance is the only signature that can distinguish convection from diffusion. In this type of diffusion the characteristic time for the non $t^{-1 / 2}$ behavior to set in would be the time required for the particles to diffuse to the boundary of the halo plus the time for the effects to propagate back to the point of observation, ie. $t_{c}=(2 D-x)^{2} / 2 \kappa$ and the spectral bend comes at $E_{c}=2 \kappa / b(2 D-x)^{2}$.

\section{COSMIC RAY NUCLEON HALO}

We have seen that the appearance of an electron halo as seen through its radio emission depends on how the actual extent of the halo $D$ compares with the characteristic distance of a diffusing/convecting medium $x_{c} \equiv \kappa / V$. Unfortunately nothing that we have discussed so far gives us an idea of the magnitude of the quantities that we need to know. It so happens that considering the effect that a dynamical halo can have on the nuclear component of the galactic cosmic rays can give us a handle on this question.

\subsection{Parker Instability}

Although the idea that a galactic halo could be important for the propagation of cosmic rays was around almost from the beginning (Bierman and Davis 1958), it wasn't until Parker's $(1965,1966,1968,1969)$ "bubble gum" picture that the notion was introduced that the cosmic rays could effect the creation of such a halo. In these papers Parker showed that the gas, magnetic field, cosmic ray system in the galactic disk was unstable. The instability occurred whenever a particular portion of a magnetic field line became slightly elevated. The cooler gas would drain away to a lower region leaving the hot, high pressure cosmic ray gas to inflate the field into an ever increasing bubble and hence escape the galactic disk. This process could be responsible for dragging the magnetic field and some of the gas out of the disk and forming a halo for the galaxy. It should be noted that this process of halo formation by its very dynamical nature necessarily produces a dynamical halo. 
This idea that the cosmic rays are responsible for driving a dynamical halo has been carried further by others the first of whom was Ipavitch (1975) who discussed a cosmic ray driven, spherical galactic wind. Others have considered this problem with more complicated geometries.

\subsection{Effect of Outflow on Disk Cosmic Rays}

Although studying the effect of the cosmic rays on the dynamics of the galactic halo is important in its own right we are most interested in examining the effect that a dynamical halo would have on the cosmic rays that we observe in the disk. It is only by studying these effects that we may hope to gain information about the dynamical properties of the halo of our galaxy.

Storage Place A galactic halo has been proposed as a storage place for cosmic rays first to produce the observed isotropy (Bierman and Davis, 1958) and later by Prischep and Ptuskin (1975) and by Ginzburg et al. (1980) to reconcile the cosmic ray age determination via measurements of unstable nuclei such as ${ }^{10} \mathrm{Be}$ with the mean grammage derived from observed secondary to primary ratios.

Aid to Lateral Diffusion Another effect that halo storage can have on the disk population of cosmic rays is that of facilitating lateral diffusion in the disk. In the case of isotropic diffusion it is intuitive that cosmic rays could not diffuse laterally to the disk much further than they can diffuse perpendicular to the disk before they escape the galaxy. In other words, a thick halo will allow cosmic-ray particles to diffuse much further from their sources during their residence time in the galaxy than will a thin one.

This idea was analyzed quantitatively by Stecker and Jones (1977) employing SAS-2 gamma ray data and CO line emission data to estimate the cosmic ray density in various regions of the disk. Assuming a source distribution similar to the supernova remnant distribution, they concluded that the halo could not be much thicker than $3 \mathrm{kpc}$. A reanalysis of this problem using COS-B data has recently been reported by Bloemen (1989) who finds a thicker halo, $\approx 20 \mathrm{kpc}$. This discrepancy appears to come from the difference between the SAS-2 and COS-B data rather from any difference in the method of analysis.

The above analysis was called into question by the work of Jokipii (1976) and Owens and Jokipii $(1977 \mathrm{a}, \mathrm{b})$ who showed that an outflowing halo could have an effect on parameters of the disk cosmic rays such as their age distribution. However, it was shown by Jones (1978) that the effect of a dynamical halo on the particles observed in the disk was to replace the actual extent of the halo with the characteristic length $x_{c}=\kappa / V$ discussed in section 2.2 .

Energy Variation of $\kappa$ is The Key It was the realization that the variation of the diffusion coefficient $\kappa$ with energy could cause a transition from diffusion dominated transport to convection dominated transport that was the key to relating cosmic ray data to parameters of the halo. The data seemed to indicate (Ormes and Freir, 
1978) that while the mean path length was a decreasing function of energy, varying as $\approx E^{-1 / 2}$ for energies above a few $\mathrm{GeV}$, below this energy the path length seemed to be constant $\approx 5-6 \mathrm{~g} \mathrm{~cm}^{-2}$. This fact would seem to indicate that $\approx 1 \mathrm{GeV}$ marks the transition between the two modes of transport.

It has been stated (Blandford and Ostriker, 1980) that if convection dominates the removal of cosmic rays from the galaxy then the escape time is independent of the energy of the particles. This is only partly true. When convection dominates the mean time spent in a fixed region of the galaxy is independent of energy, however, mean age of a particle is given by $t_{c} \approx \kappa / V^{2}$ and is therefore, dependent upon energy through the diffusion coefficient, $\kappa$. During this mean lifetime the particle has been confined to a distance $x_{c}=\kappa / V$ on either side of the disk and thus the amount of time spent in a disk of thickness $a$ is given by $t_{c} a / x_{c}=a / V$ which is independent of $\kappa$ and hence independent of energy.

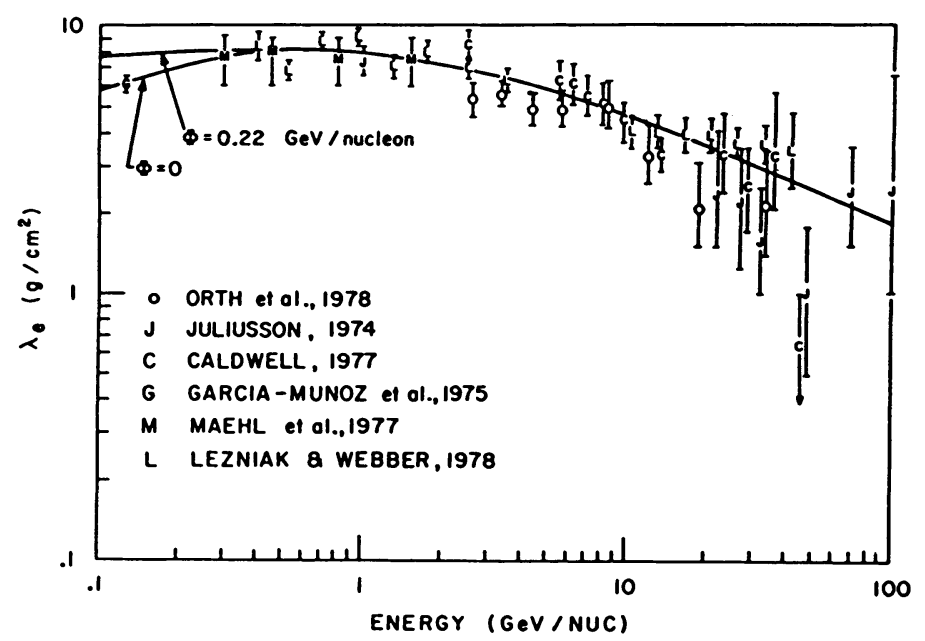

Figure 4. Mean Path Length vs. Energy for Cosmic Rays at Earth

Dynamical Halo Model of Our Galaxy Analysis of this situation by Jones (1979), Freedman et Al. (1980), and Kota and Owens (1980) indicated that the data could be fit by a dynamical halo model with a wind speed of $8-20 \mathrm{~km} \mathrm{~s}^{-1}$ the particular value depending on the value of the disk thickness in $\mathrm{g} \mathrm{cm}^{2}$ assumed by the authors. Although the particular values of $\kappa$ and the size of the halo were much harder to obtain due to the uncertainty of the actual mean age of the cosmic rays. The main point of the analysis can be seen from Figure 4 and that is that for energies above $1 \mathrm{GeV}$ per nucleon or rigidities above $3.4 \mathrm{GV}$ the galactic halo of our galaxy is diffusion dominated. This is true if the low energy flattening of the path length vs. energy curve is due to a dynamical halo regardless of the numerical value of any quantity derived from the theory. 


\section{CONCLUSION}

This, of course, implies that the transport electrons above 3 or $4 \mathrm{GeV}$ is also diffusion dominated and that, assuming a magnetic field of $1 \cdot 10^{-6} \mathrm{~g}$ in the halo, no obvious convection effects can be seen at frequencies above about $100 \mathrm{MHz}$. One must look for the spectral cut off that represents the first arrival time of the electrons at the point of observation and try to determine whether this scales with the inverse square of the distance from the disk or as the simple inverse. In addition one may identify a bend in the spectrum but realizing that this is not an unambiguous sign of convection one must try to determine its behavior as a function of position in the halo.

I believe that convection effects can be detected in radio halos but it will not be done by identifying some single feature in the spectrum. Rather one will have to map out the halo region and find how spectral features move from one place to another to determine whethte the move adout by convection or diffusion.

\section{REFERENCES}

Bierman, J. L., Davis, L. (1958) Zs. Ap. 51, 19

Blandford, R. D., Ostriker, J. P. (1980) Ap. J. 237, 793

Bloemen, J. B. G. M. (1989) Adv. Space Res. 10, (2)199

Freedman, I., Giler, M., Kearsey, S., Osborne, J. L. (1980) Astron. Ap. 82, 110

Ginzburg, V. L.,Khazan, Ya. M., Ptuskin, V. S. (1980) Ap. Space Sci. 68, 295

Ipavitch, F. M. (1975) Ap. J. 196, 107

Jokipii, J. R. (1976) Ap. J. 208, 900

Jones, F. C. (1978) Ap. J. 222, 1097

Jones, F. C. (1979) Ap. J. 229, 747

Kota, J., Owens, A. J. (1980) Ap. J. 237, 814

Lerche, I., Schlickeiser, R. (1980) Ap. J. 239, 1089

Lerche, I., Schlickeiser, R. (1981a) Ap. J. Suppl. 47, 33

Lerche, I., Schlickeiser, R. (1981b) Astron. Ap. 107, 148

Lerche, I., Schlickeiser, R. (1981c) Ap. Lett. 22, 31

Owens, A. J., Jokipii, J. R. (1977a) Ap. J. 215, 677

Owens, A. J., Jokipii, J. R. (1977b) Ap. J. 215, 685

Parker, E. N. (1965) Ap. J. 142, 584

Parker, E. N. (1966) Ap. J. 145, 811

Parker, E. N. (1968) in Stars and Stellar Systems, Vol. VII: Nebulae and Interstellar Matter, eds. B. M. Middlehurst, L. H. Allen, University of Chicago Press, Chicago, Chapt. 14

Parker, E. N. (1969) Space Sci. Rev. 9, 651

Pikel'ner, S. B. (1953) Doklady Akad. Nauk SSSR 88, 229

Prischep, V. L., Ptuskin, V. S. (1975) Ap. Space Sci. 32, 265

Shklovsky, I. S. (1952) Astr. Zh. 29, 418

Stecker, F. W., Jones, F. C. (1977) Ap. J. 217, 843 